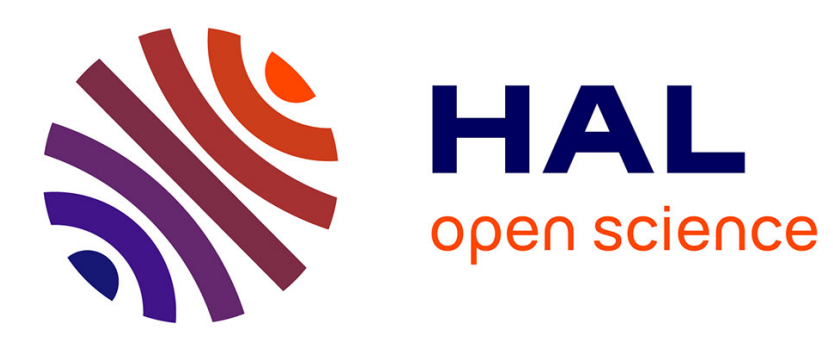

\title{
Characterization of triboelectrically charged particles deposited on dielectric surfaces
}

\author{
A Nesterov, F Löffler, Yun-Chien Cheng, G Torralba, K König, M Hausmann, \\ V Lindenstruth, V Stadler, F R Bischoff, F Breitling
}

\section{- To cite this version:}

A Nesterov, F Löffler, Yun-Chien Cheng, G Torralba, K König, et al.. Characterization of triboelectrically charged particles deposited on dielectric surfaces. Journal of Physics D: Applied Physics, 2010, 43 (16), pp.165301. 10.1088/0022-3727/43/16/165301 . hal-00569582

\section{HAL Id: hal-00569582}

\section{https://hal.science/hal-00569582}

Submitted on 25 Feb 2011

HAL is a multi-disciplinary open access archive for the deposit and dissemination of scientific research documents, whether they are published or not. The documents may come from teaching and research institutions in France or abroad, or from public or private research centers.
L'archive ouverte pluridisciplinaire HAL, est destinée au dépôt et à la diffusion de documents scientifiques de niveau recherche, publiés ou non, émanant des établissements d'enseignement et de recherche français ou étrangers, des laboratoires publics ou privés. 


\title{
Characterization of Triboelectrically Charged Particles Deposited on Dielectric Surfaces
}

\author{
A Nesterov ${ }^{2,3}$, F Löffler ${ }^{1,2}$, Yun-Chien Cheng ${ }^{1}$, G Torralba ${ }^{2}$, K König ${ }^{1,2}$, M Hausmann $^{2}$, \\ V Lindenstruth ${ }^{2}$, V Stadler ${ }^{1}$, F R Bischoff ${ }^{1}$ and F Breitling ${ }^{3}$ \\ ${ }^{1}$ German Cancer Research Centre, In Neuenheimer Feld 280, Heidelberg, Germany, \\ ${ }^{2}$ Kirchhoff Institute of Physics, In Neuenheimer Feld 227, Heidelberg, Germany; \\ ${ }^{3}$ Karlsruhe Institute of Technology (KIT), Institute for Microstructure Technology, \\ Herrmann von Helmholtzplatz 1, 76344 Eggenstein-Leopoldshafen, Germany; Frank.Breitling@ KIT.edu; \\ alexander.nesterov-mueller@kit.edu
}

\begin{abstract}
A device for the measurement of $\mathrm{q} / \mathrm{m}$-values and charge degradation of triboelectrically charged particles deposited on a surface was developed. The setup is based on the integration of currents, which are induced in a Faraday cage by insertion of a solid support covered with charged particles. The conductivity of different particle supports was taken into account. The "blow-off" method, in which the particles are first deposited, and then blown off using an air stream, can be used for characterisation of triboelectric properties of particles relative to different surfaces.
\end{abstract}

PACS: 85.85.+j, 87.80.Ek

\section{Introduction}

Triboelectric charge is an important characteristic of nano and micro particles. Particles of such size can exhibit $\mathrm{q} / \mathrm{m}$-values ( $\mathrm{q}$ is the particle charge, $\mathrm{m}$ is the mass) above $10^{-3} \mathrm{C} / \mathrm{kg}$, which can significantly influence their dynamics. Particle charges of triboelectric origin play an important role both in natural processes such as dune movement [1], nucleation [2], aerosol-cloud interaction [3] and in applications, for example in electrophotography [4], triboelectrostatic separation [5], combinatorial chemistry [6], etc. Particles can acquire their charge in the manufacturing process or during transport due to contact with various surfaces.

Different methods exist for particle charge measurement. Mass spectrometric methods exploit particle deflection in an electrical field [7-9]. The particle charge is calculated from the balance of forces acting on the particles. Other methods are based on the use of a Faraday cage. In air, moving particles are absorbed in the Faraday cage and the induced charge is measured [9]. As in the case of the mass spectroscopic method, only a single measurement for the same particles is possible, i.e. the particles are not amenable for new measurements after their deflection or absorption. A cylindrical probe can also act as a Faraday cage [10]. The particles can move through the cylindrical probe without being absorbed. In this case, the charge of the same particles can be measured only twice: at the inlet and the outlet of the probe.

Particle charging can be controlled by placing an inductive probe directly into the charging chamber [11]. In this case, the particles are constantly in motion and are charged by contacting the walls of the charging chamber or other particles. If the particles do not move, no signal is induced in the probe. Hence, it is rather complicated to measure the charge of the same particles over time with the methods mentioned above. 
In this article, triboelectrically charged particles deposited on a dielectric surface are studied. This aspect is interesting from the following point of view: On solid surfaces,
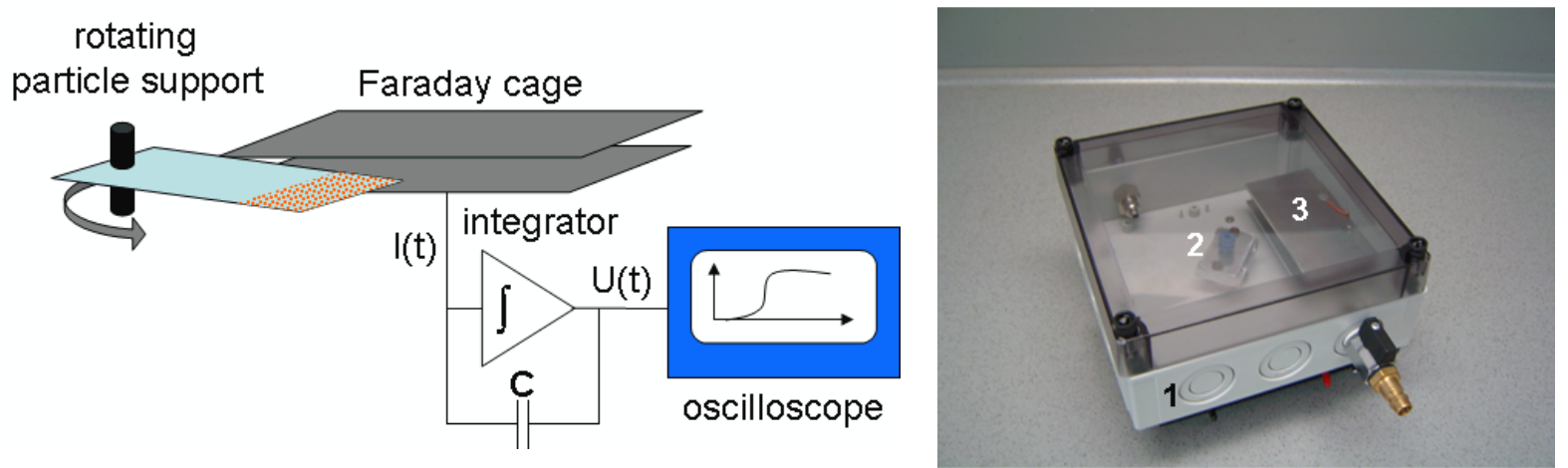

Fig. 1: Schematic drawing of the experimental setup used to measure the charge of particles deposited on a particle support (left) and a photo of the tribometer (right): housing (1), rotating particle support holder (2), Faraday cage (3).

particles can usually be manipulated in a more predictable way than in a gas stream. In the two phase stream, particles generally exhibit a very broad velocity distribution and their trajectories are complicated because of
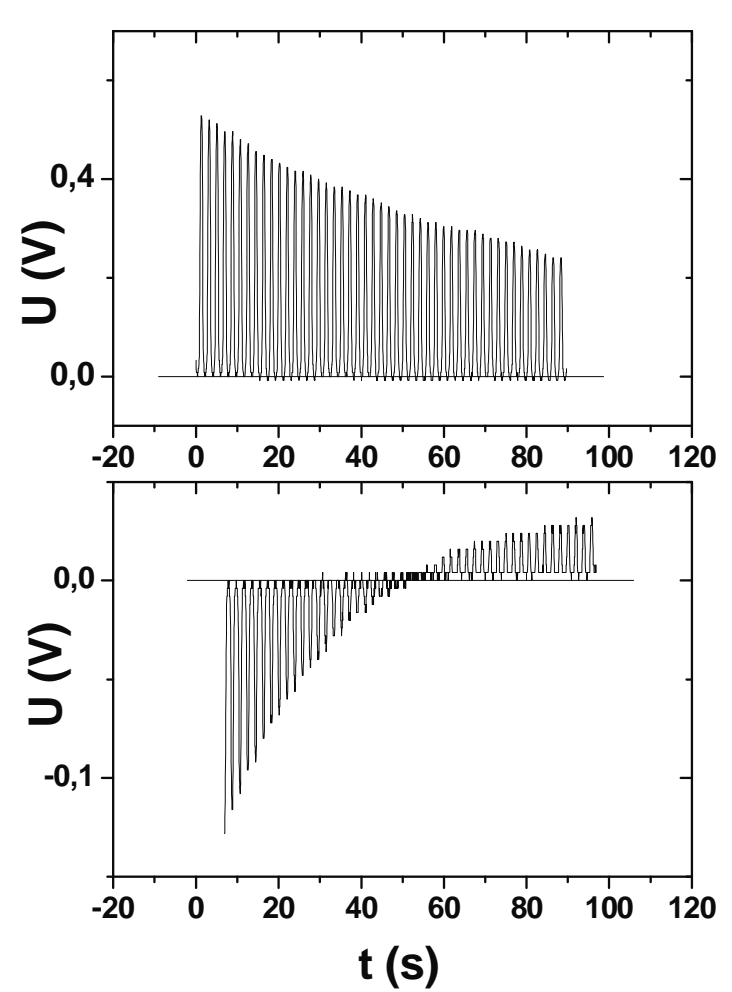

Figure 2: Examples of measured voltages over time: (top) charge degradation; (bottom) inversion of charge polarity. The particle charge is calculated using $Q(t)=$ $\mathrm{CU}(\mathrm{t})$. The maxima of the plot correspond to the particle position inside the Faraday cage; the minima of the absolute voltage (zero in the ideal case) correspond to the complete removal of the particle support out of the Faraday cage. turbulences. On the other hand, particles on a solid support have the same velocity as their support and can be located at defined distances with a high precision. These features could be developed into advantages in applications like combinatorial chemistry where particle deposition with a high spatial resolution and minimized background is necessary.

\section{Experimental setup - tribometer}

\subsection{Tribometer}

The outline of the experimental setup - called tribometer - is displayed in figure 1: a particle support with triboelectrically charged particles is periodically inserted into the Faraday cage. In the tribometer, a particle support rotates with a frequency of 0.3 $\mathrm{Hz}$. The metal cage is fabricated in a way that it does not prohibit this rotation. It works like Faraday cup shielding the electrical field of particles on the support. In this process, a current is induced in the Faraday cage, which is integrated by the electronic circuit shown in the figure 1 . The integrated value is the voltage $U$, which is measured with an oscilloscope. The voltage $U$ displayed on the oscilloscope is related to the particle charge $Q$ through the capacity $C$ with: $Q=-C U$. Figure 2 shows an example of an $\boldsymbol{U}(\mathrm{t})$ function measured as 
outlined in figure 1. The maxima of the plot correspond to a position of the particles on their support inside of the Faraday cage, the smallest absolute voltages (zero in the ideal case) correspond to the complete removal of the particle support out of the cage.

The total mass $m$ of particles is obtained by weighing the particle support with and without the particles. Subsequently, the $\mathrm{q} / \mathrm{m}$-value is calculated.

For measurements with the tribometer, it is important that the total charge of the particle support is significantly smaller than the particle charge. The tribometer is useable both for micro and nano particles. The sensitivity of the tribometer can be adjusted by tuning the capacitance $C$. $C=100 \mathrm{nF}$ was chosen for the measurements described here.

The functionality of the tribometer was examined with different types of micro particles: laser printer toner particles (OKI) with an average diameter of $10 \mu \mathrm{m}$ [12], laser printer toner particles (Canon) with an averaged diameter of $5.5 \mu \mathrm{m}$ [13] and laboratory-made polymer amino acid particles (AAP) [14] with diameters larger than $45 \mu \mathrm{m}$. This size distribution was generated using a Retsch $45 \mu \mathrm{m}$ mesh analytical sieve.

Microscope glass slides, Polystyrol and PVC plates are used as particle supports. All particle supports possess a standard microscope slide area format of $7.5 \mathrm{~mm} \times 2.5 \mathrm{~mm}$. The thickness of microscope slides is $1.1 \mathrm{~mm}$. The thickness of Polystyrol and PVC plates is $1.5 \mathrm{~mm}$.

\subsection{Aerosol chamber}

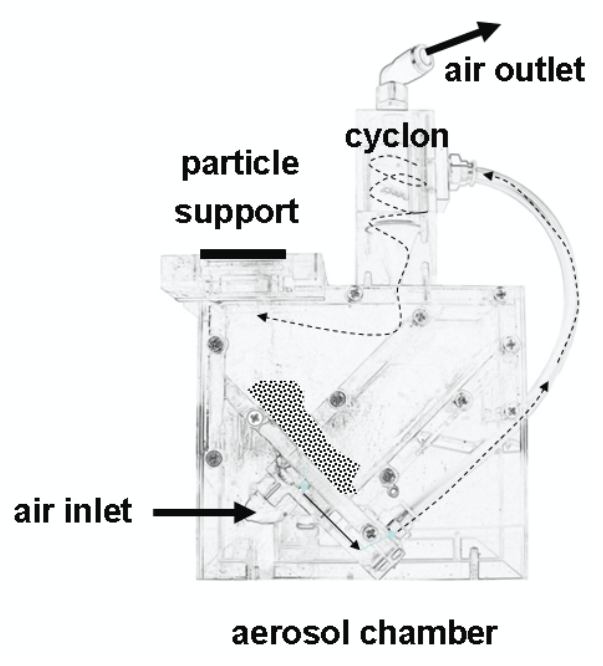

Figure 3: Aerosol chamber

An air stream at room temperature is used to deposit particles on a particle support. A plexiglas chamber was fabricated to conduct the particle deposition (Fig. 3). The chamber additionally fulfils two functions: destruction of particle agglomerates and particle triboelectric charging.

The particles are sucked up by the air stream at the bottom of the reservoir chamber and conveyed through a separate channel into the cone chamber for triboelectric charging. Uncharged particles possess $\mathrm{q} / \mathrm{m}$-values below $10^{-4} \mathrm{C} / \mathrm{kg}$. The inlet of the cone chamber is shifted so that the particles move along the spiral trajectories touching the chamber walls. The conglomerates are disrupted by this movement. Afterwards, the particles again pass into the reservoir chamber and reach the chip surface through the aperture. The particle layer was not strictly homogenous in our case. OKI and Canon particles formed a layer with a thickness of ca. 15$30 \mu \mathrm{m}$. AAP particles $(45 \mu \mathrm{m})$ formed predominately one particle layer. Our experiments were conducted in the air-conditioned particle room with humidity about $28 \%$. The support area $\mathrm{S}$ deposited with particles was $18 \times 18 \mathrm{~mm}^{2}$. The total charge Q was measured with the tribometer. The charge density was calculated as a relation $\mathrm{Q} / \mathrm{S}$.

\section{Results and discussion}

\subsection{Charge of particle supports}

The charge of appropriate particle supports was studied with the tribometer. As a rule, such supports carry a charge because of their contacts with other objects: holders, gloves etc. To minimize this charge, we washed the supports with ethanol and blew them off with the compressed air. Just after blowing off, the supports induced voltages $\boldsymbol{U}$ below $5 \mathrm{mV}$ in the tribometer, which corresponds to $Q=5 \times 10^{-10} \mathrm{C}$. 
After 1 minute of handling, the voltage and respectively the charge, regenerates and reaches stable values. These values are shown in table 1 for different supports used in our experiments. All supports used showed a negative surface charge density. The manufacturers of microscope glass slides Diapath and Menzel report that their slides are covered with a positive electrostatic film to enhance the adsorption of cytological tissues $[15,16]$. The reason for the registered negative stable charge will be discussed in the next paragraph.

Table 1. Measured stable values for different particle supports:

\begin{tabular}{|l|c|c|}
\hline particle support material & $\begin{array}{c}\text { base value, } \\
{[\mathbf{m V}]}\end{array}$ & $\begin{array}{c}\text { mean charge density, } \\
\mathbf{1 0}^{-6}\left[\mathbf{C}^{\mathbf{2}}\right]\end{array}$ \\
\hline microscope glass slide Menzel [15] & $\mathbf{- 1 2} \pm \mathbf{2}$ & $\mathbf{- 1 , 9 2}$ \\
\hline microscope glass slide Diapath [16] & $-\mathbf{1 2} \pm \mathbf{2}$ & $\mathbf{- 1 , 9 2}$ \\
\hline microscope glass slide R. Langenbrinck [17] & $-\mathbf{6} \pm \mathbf{2}$ & $\mathbf{- 0 , 9 6}$ \\
\hline Polystyrol & $-\mathbf{6} \pm \mathbf{2}$ & $\mathbf{- 0 , 9 6}$ \\
\hline PVC & $\mathbf{- 8} \pm \mathbf{2}$ & $\mathbf{- 1 , 2 8}$ \\
\hline
\end{tabular}

\subsection{Influence of the surface}

Triboelectrically charged particles were deposited on the particle supports described in table 1 and tested with the tribometer. Three qualitatively different results were observed. A stable signal was measured on polystyrene and polyvinyl chloride (PVC) surfaces. The charge of the particles on the microscope glass slides R. Langenbrinck [17] was below the device sensitivity. Charge degradation was observed on the particle supports Menzel and Diapath.

Figure 4 shows the degradation curves of $\mathrm{q} / \mathrm{m}$-values for the microscope glass slides Menzel and Diapath covered with Canon- and OKI- laser printer particles. As one can see, the charge degradation does not significantly depend on the particle size and composition and obeys an exponential law. The tendency of triboelectrified particles to follow exponential

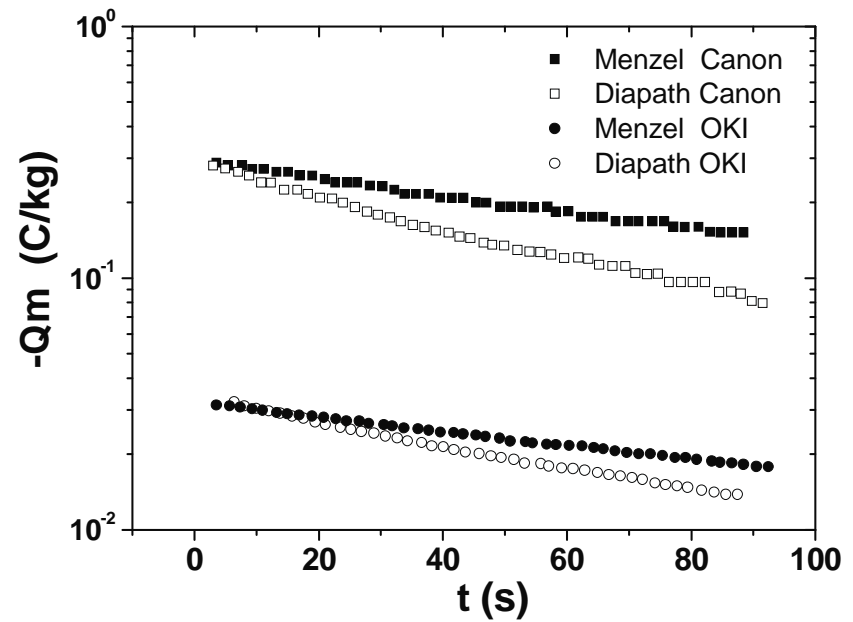

Figure 4: Degradation of $q / \mathrm{m}$-values for OKI and Canon particles, measured on different microscope slides by Menzel [15] and Diapath $[16]$. and the conductive surface. As expected, the charge degradation occurs faster for shorter distances between the particle and the conducting path.

The conductivity could be used to explain the stable negative charge values of glass microscope slides discussed in 3.1. They have the same potential as the slide holder. 
The microscope glass slides R. Langenbrinck

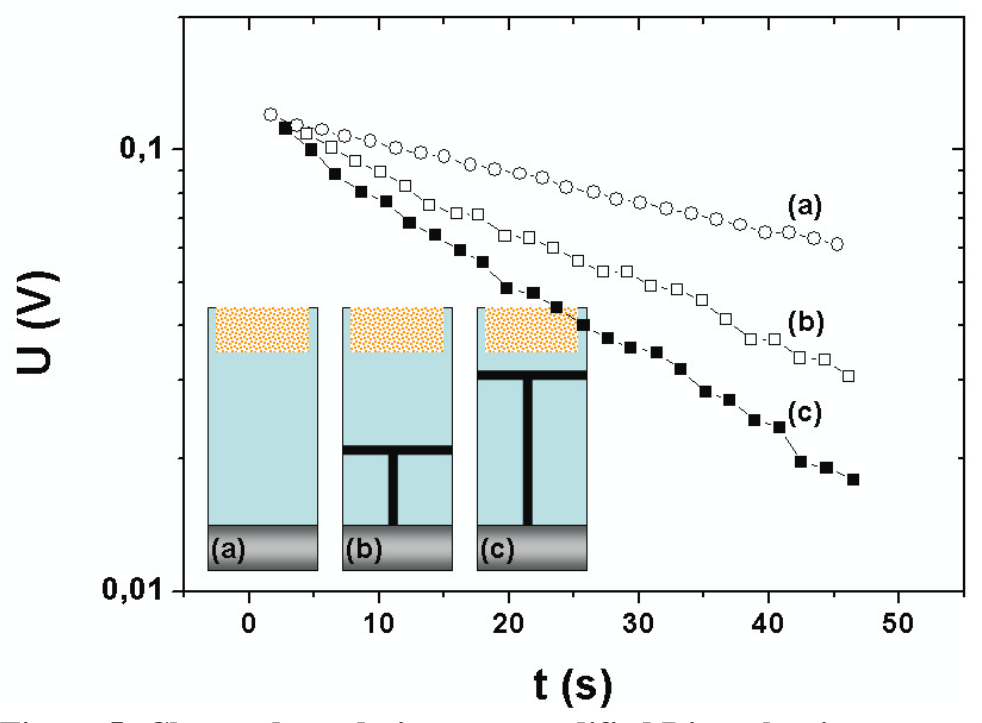

Figure 5: Charge degradation on a modified Diapath microscope glass slide for Canon particles. Microscope slide (a) is unmodified. On microscope slides (b) and (c), a conductive glue was attached as shown.

contacts.

Nevertheless, one can use microscope glass slides like Menzel und Diapath slides for measuring particle $\mathrm{q} / \mathrm{m}$-values. The $\mathrm{q} / \mathrm{m}$-values are obtained by interpolation of the degradation line to the point $\mathrm{t}=0 \mathrm{~s}$, at which the slide contacted the holder provided that the exponential relation is valid. The $\mathrm{q} / \mathrm{m}$-values $-0.27 \mathrm{C} / \mathrm{kg}$ for Canon particles and $-0.031 \mathrm{C} / \mathrm{kg}$ for OKI particles are obtained at beginning of the degradation curves in figure 4 . These values correspond to the following surface charge densities $\sigma=-1.35 \times 10^{-3} \mathrm{C} / \mathrm{m}^{2}$ for Canon particles and $\sigma=-3.1 \times 10^{-4} \mathrm{C} / \mathrm{m}^{2}$ for OKI particles, assuming that the mass density equals $1 \mathrm{~g} / \mathrm{cm}^{3}$ for both types of the particles. Obviously, the exponential law is valid for surface charge densities which are about 2 decades greater than the critical surface charge density.

\subsection{Search for air ions}

Some authors pointed out that a discharging process should be taken into account

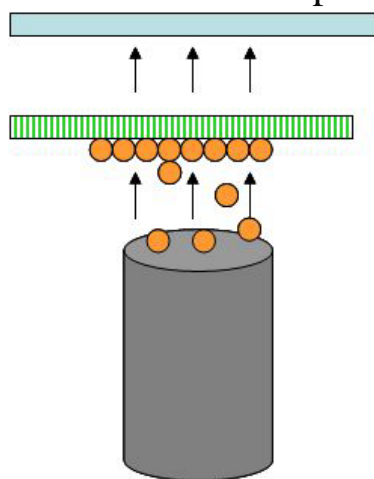

Figure 6: Schematic of the set up to search for ion generation by the triboelectric charging. AAPparticles with diameters larger than $45 \mu \mathrm{m}$ were used. The mesh size of the sieve is $10 \mu \mathrm{m}$. when considering the triboelectric charging [22-24]. We tried to use the tribometer to find proof for or against the hypothesis that triboelectric charging is accompanied by the generation of air ions. Actually, this effect could look like corona discharging [4]. The electrical field between two surfaces is proportional to their electric potential difference and inversely proportional to the distance between them. Hence, in a triboelectric contact, electric fields can be easily larger than the critical discharging field in the air.

A nylon sieve with a mesh size of $10 \mu \mathrm{m}$ (Franz Eckert $\mathrm{GmbH}$ ) was placed between the microscope slide and the air stream with triboelectrically charged AAP-particles as shown in figure 6 . Thus, only carrier gas without particles could contact the microscope slide. No charging of the microscope slide above a noise of $0.8 \times 10^{-6} \mathrm{C} / \mathrm{m}^{2}$ was observed in this experiment. One may need a more sensitive 
technique for studying discharging effects by triboelectric charging of particles.

\subsection{Triboelectric charging of surfaces by blowing off particles}

Figure 6 shows degradation curves obtained in the following experiment. First, the triboelectrically charged particles were deposited on a slide and the degradation of the resulted negative charge was measured with the tribometer as in the previous cases (Fig. 7, group I). Then, the particles were blown off from the slide using compressed air at a velocity of approx. $50 \mathrm{~m} / \mathrm{s}$. The resulted charge of the slide surfaces was measured. By blowing off the particles, a positive charge was obtained on the slides (degradation curves in group II, Fig 7).

Three sorts of particles were used

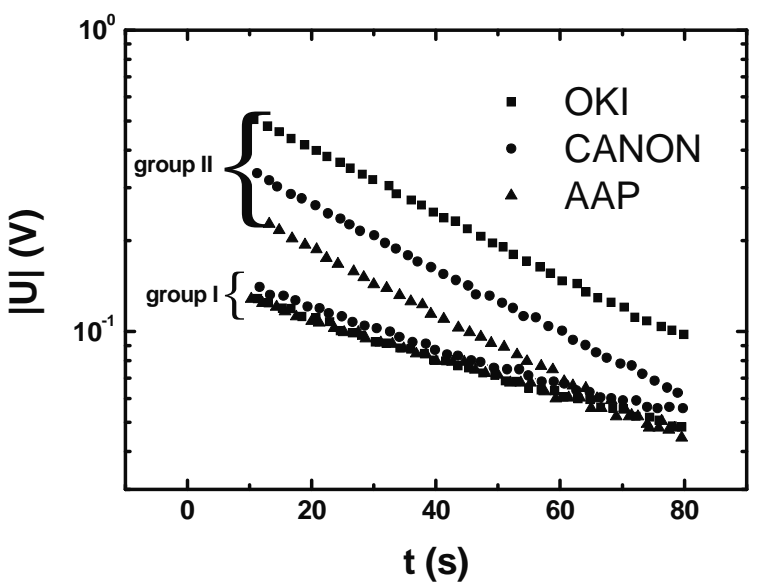

Figure 7: Charge degradation curves on a Menzel microscope slide. Three particle sorts are used. The curves from the group I correspond to degradation of the negative charge on the microscope slides with deposited particles. The curves from the group II correspond to degradation of the positive charge generated on the microscope slides after blowing away the particles.
(Canon, OKI, AAP).

The absolute values of the charge obtained by removal of the particles from slides are larger than the initial triboelectric charge of the same particles. This shows that the particles can be very efficiently triboelectrically charged. The surface charge density of the slides in the case of the OKI particles was $\sigma=7.8 \times 10^{-5} \mathrm{C} / \mathrm{m}^{2}$.

This experiment confirms our earlier statement that the degradation occurs according to the exponential law and degradation time constants are independent of particle size and composition. In our case, the degradation time constant for positive charge is larger than that for the negative charge.

Consequently, the mobility of negative charge carriers involved in the surface charge transport is greater than the mobility of positive charge carriers.

The fact that particles can be relatively highly charged by removal from a surface alone should be taken into account by particle manipulation. Particle removal from surfaces takes place in practically all apparatus. It is very difficult to predict the particle charge theoretically because of the complex character of the triboelectric effect. In contrary, the proposed "blow off" method based on the tribometer allows for easier experimental characterisation of particle triboelectrification relative to different surfaces.

\section{Conclusion}

Our objective was to study the possibility of characterizing triboelectrically charged particles deposited on surfaces. Additionally, this idea gives the answer to how a device for the characterization should be constructed. Such a set up, called tribometer, is based on the integration of induced currents, which appear by inserting the support with charged particles into a Faraday cage.

Particle $\mathrm{q} / \mathrm{m}$-values can be measured using the tribometer. A particle support with a relative surface conductivity as PVC, polystyrene or different microscope glass slides can be used for these measurements. One should always ensure that the charge of the particle support does not influence the measurements. 
Our experiments have shown that conductivity of microscope slides from different manufactures varies. Some microscope slides can be charged or discharged by a conductive contact within few minutes.

The "blow-off" method has been used for the characterization of triboelectric properties of particles relative to different surfaces. Exponential degradation of charges was confirmed.

\section{Acknowledgment}

We thank D. Gottschalk for the circuit design of the device and Dr. S. Fernandez for manufacturing the amino acid particles. We thank Ministry of Education, Taiwan for the financial support. We acknowledge financial support from the Landesstiftung Baden-Wuerttemberg. 


\section{References:}

[1] Kok J F and Renn N O 2008 Electrostatics in Wind-Blown Sand. Phys. Rev. Lett. 014501

[2] Winkler P M, Steiner G M, Reischl G P, Vrtala A, Wagner P E and Kulmala M 2008 The Effect of Seed Particle Charge on Heterogeneous Nucleation, in Nucleation and Atmospheric Aerosols, Springer

[3] Harrison R G 1997 Climate changes and the global atmospheric electrical system. Atmos. Environ. 31 3483-84.

[4] Pai D M and Sprigett B E 1993 Physics of electrophotography. Rev. Mod. Phys. 65 163211

[5] Stencel J M, Schaefer J L, Heng Ban and Neathery J K 1999 Apparatus and method for triboelectrostatic separation, US Patent 5938041

[6] Beyer M, Nesterov A, Block I, König K, Felgenhauer T, Fernandez S, Leibe K, Torralba G, Hausmann M, Trunk U, Lindenstruth V, Bischoff F R, Stadler V and Breitling F 2007 Combinatorial Synthesis of Peptide Arrays onto a Microchip. Science 3181888

[7] Inculet I I and Castle G S E 1991 Tribo-electrification of commercial plastics in air, Pro. 8th International Conf. on Electrostatics ELECTROSTATICS '91, Oxford, England, in: Inst. Phys. Conf. Set. No. 118 217-222.

[8] Hogue M D, Calle C I, Curry D R, Weitzman P S 2009 Discrete element modelling (DEM) of triboelectrically charged particles: Revised experiments, Journal of Electrostatics 67, 691-694.

[9] Trigwell S, Tennal K B, Mazumder M K, Lindquist D A 2003 Precombustion cleaning of coal by triboelectric separation of minerals. Particulate Science Technology 21353 364

[10] Nesterov A, Löffler F, König K, Trunk U, Leibe K, Felgenhauer T, Bischoff F R, Breitling F, Lindenstruth V, Stadler V and Hausmann M 2007 Measurement of triboelectric charging of moving micro particles by means of an inductive cylindrical probe. J. of Phys. D Appl. Phys. 40 6115-20

[11] Smolders A, Baeyens J 1992 Continous monitoring of particulate emissions by means of triboelectric probe. Powder 9 (2) 123 - 127

[12] http://www.oki-osd.de

[13] http://www.usa.canon.com

[14] Beyer M, Nesterov A, Block I, König K, Felgenhauer T, Fernandez S, Leibe K, Torralba G, Hausmann M, Trunk U, Lindenstruth V, Bischoff F R, Stadler V and Breitling F 2007 Combinatorial Synthesis of Peptide Arrays onto a Microchip, Science 3181888 Supporting online material

[15] http://www.menzel.de

[16] http://www.diapath.com

[17] http://www.langenbrinck.com

[18] Greason W D 2000 Investigation of a test methodology for triboelectrification, Journal of Electrostatics 49 (3-4) 245-256

[19] Hogue M D, Calle C I, Weitzman P S, Curry D R, 2008 Calculating the trajectories of triboelectrically charged particles using Discrete Element Modeling (DEM), Journal of Electrostatics, 66 32-38

[20] Kindersberger J, Lederle Ch 2008 Surface Charge Decay on Insulators in Air and Sulfurhexafluorid. IEEE Transactions on Dielectrics and Electrical Insulation 15 94147

[21] Ieda M, Sawa G and Shinohara U 1968 Decay of electric charges on polymer films, Electrical Engineering in Japan 88 67-73 
[22] Charlson E M, Charlson E J, Burkett S and Yasuda K H 1992 Study of the contact electrification of polymers using contact and separation current. IEEE Transactions on Electrical Insulation 271144 - 51

[23] Lee L-H 1994 Dual mechanism for metal polymer contact electrification. J. Electrostat. 32 1-29

[24] Lowell J 1979 Tunneling between metals and insulators and its role in contact electrification. J. Phys. D: Appl. Phys. 121541 - 54 


\section{Figure Captures}

Figure 1: Schematic drawing of the experimental setup used to measure the charge of particles deposited on a particle support (a) and a photo of the tribometer (b): housing (1), rotating particle support holder (2), Faraday cage (3).

Figure 2: Examples of measured voltages over time: (top) charge degradation; (bottom) inversion of charge polarity. The particle charge is calculated using $\mathrm{Q}(\mathrm{t})=-\mathrm{CU}(\mathrm{t})$. The maxima of the plot correspond to the particle position inside the Faraday cage; the minima of the absolute voltage (zero in the ideal case) correspond to the complete removal of the particle support out of the Faraday cage.

Figure 3: Aerosol chamber

Figure 4: Degradation of $\mathrm{q} / \mathrm{m}$-values for OKI and Canon particles, measured on different microscope glass slides by Menzel [16] and Diapath [17].

Figure 5: Charge degradation on a modified Diapath microscope slide for Canon particles. Microscope glass slide (a) is unmodified. On microscope slides (b) and (c) conductive glue was attached as shown.

Figure 6: Schematic of the set up to search for ion generation by the triboelectric charging. AAP-particles with diameters larger than $45 \mu \mathrm{m}$ were used. The mesh size of the sieve is 10 $\mu \mathrm{m}$.

Figure 7: Charge degradation curves on a Menzel microscope glass slide. Three particle sorts are used. The curves from the group I correspond to degradation of the negative charge on the microscope slides with deposited particles. The curves from the group II correspond to degradation of the positive charge generated on the microscope slides after blowing away the particles.

Table 1: Measured stable values for different particle supports: 